\title{
Experimental Evaluation of Unipolar OFDM VLC System on Software Defined Platform
}

\author{
Bassam Aly*†, Mohammed Elamassie ${ }^{\dagger}$, Murat Uysal ${ }^{\dagger}$, and Emrah Kınav* \\ ${ }^{*}$ R\&D Department, Ford Otosan, Istanbul, Turkey, 34885. \\ ${ }^{\dagger}$ Department of Electrical and Electronics Engineering, Özyeğin University, Istanbul, Turkey, 34794. \\ E-mails: bmohamed@ford.com.tr, mohammed.elamassie@ozu.edu.tr, murat.uysal@ozyegin.edu.tr, ekinav@ ford.com.tr.
}

\begin{abstract}
With unique features and advantages, visible light communication (VLC) is considered as a powerful complementary wireless access technology to radio frequency solutions such as WiFi. To handle the frequency-selectivity of VLC channels particularly at high speeds, multi-carrier signaling in the form of orthogonal frequency division multiplexing (OFDM) is typically preferred. Among various variants of optical OFDM, unipolar OFDM (U-OFDM) stands outs with its better error rate performance. In this paper, we present the experimental evaluation of the bit error rate (BER) performance of a $U$ OFDM-based VLC system. In our experimental set-up, we use the popular software defined platform USRP (Universal software radio peripheral) from National Instruments customized with baseband cards and integrated with VLC front-ends. We first characterize the frequency-selective VLC channel. Then, we present BER performance against the estimated signal-to-noiseratio and confirm it through comparison with theoretical results.
\end{abstract}

Index Terms-Visible light communication, software defined, USRP, unipolar orthogonal frequency division multiplexing.

\section{INTRODUCTION}

Visible light communication (VLC), also known as LiFi, is based on the principle of modulating the light intensity of light emitting diodes (LEDs) [1], [2]. This lets the dual use of LEDs for both illumination and communication purposes. In a VLC system, the information-carrying signal is imposed as an AC signal on the LED drive current. Since the frequency of AC signal is high enough, flicker is invisible for the human eye. There is also no adverse effect on the illumination level for a DC-balanced modulating signal with a constant average value. VLC is mainly positioned as a complementary wireless access technology to radio frequency solutions (such as WiFi) for data off-loading. VLC provides higher spectral area efficiency due to frequency reuse and is particularly attractive for user-dense environments. Spatial confinement of VLC further provides an inherently secure solution.

In a VLC system, amplitude constraints should be considered in the selection of modulation technique to ensure the non-negativity of the signal. Taking into account this, the

The work of Bassam Aly was supported by the European Horizon 2020 MSC ITN (VISION) under Grant 764461. The work of M. Uysal was supported by the Turkish Scientific and Research Council (TUBITAK) under Grant 215E311. earlier works on VLC mainly focused on pulse modulation techniques such as on-off keying (OOK), pulse position modulation (PPM) and pulse amplitude modulation (PAM) [3]. To handle the frequency-selectivity of VLC channels particularly at high speeds, more recent works are built upon various forms of orthogonal frequency division multiplexing (OFDM) modified to obtain a real valued and unipolar signal suitable for driving the light source [3].

Hermitian symmetry is typically used in optical OFDM schemes to make the signal real valued at the transmitter side. To obtain a unipolar signal, various techniques are used. In DC-biased optical OFDM (DCO-OFDM) [4], a DC bias is simply added to shift the bipolar signal into the dynamic range of LED. In asymmetrically-clipped optical OFDM (ACOOFDM) [5], only odd subcarriers are modulated; this ensures a symmetry in time domain and makes it possible to clip the non-negative values without any loss of information. Another alternative is unipolar OFDM (U-OFDM) [6], also known as Flip-OFDM [7], where each time sample is encoded into a pair of new time samples. For instance, if the sample is positive valued, it is encoded to positive of the magnitude followed by zero. On the other hand, if the sample is negative valued, it is encoded as a zero followed by the positive magnitude value of the negative sample. By grouping the first samples of each pair, we can obtain the so-called "positive OFDM sub-frame". Similarly, by grouping the second samples of each pair, we can obtain the "negative OFDM sub-frame". The U-OFDM signal is obtained by concatenating positive and negative sub-frames. At the receiver side, the decoding process is simply achieved by subtracting the negative sub-frame from the positive one. It was reported in [6] that U-OFDM is more power efficient than DCO-OFDM and gives better error rate performance than both DCO-OFDM and ACO-OFDM.

While initial works on U-OFDM were based on theoretical investigations and simulations, see e.g., [6]-[8], some experimental evaluations of U-OFDM were also reported in [9], [10]. In [9], laboratory measurement/analysis equipment are used where the signal is generated through an arbitrary waveform generator and the received signal is processed offline in MATLAB. In [10], transmitters and receivers are designed based on pure hardware solutions such as field programmable gate array (FPGA). 


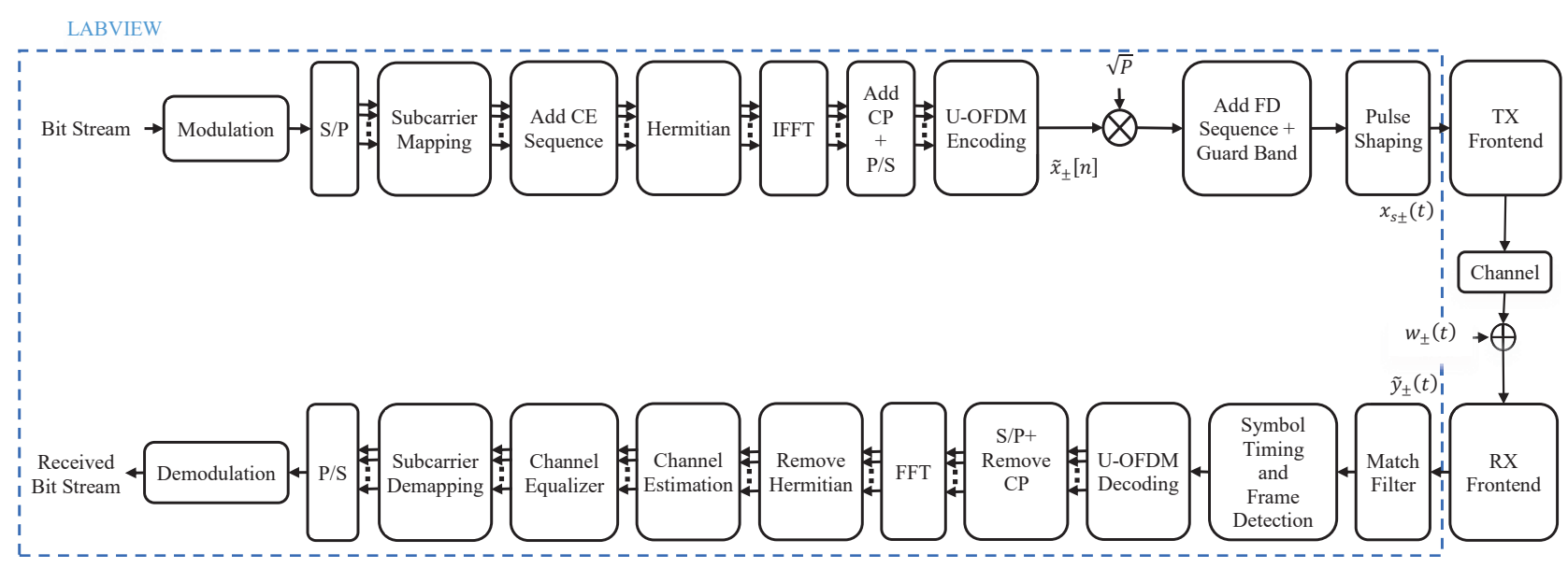

Fig. 1. Block diagram of U-OFDM system under consideration

As a flexible alternative, software defined platforms can be used, where physical layer functionalities are implemented through modifiable software or firmware. Some experimental studies on VLC using software defined platforms were reported in [11]-[15]. For example, in [14], the popular software defined platform USRP (Universal software radio peripheral) from National Instruments (NI) was modified and integrated with a VLC front-end to experimentally evaluate the performance of several physical layer modes (i.e., Manchester encoded OOK and 4B6B encoded PPM) of IEEE 802.15.7 standard. Some experimental results on ACO-OFDM and DCO-OFDM with software defined platforms can be found in [12], [15]. To the best of our knowledge, implementation of UOFDM on a software defined platform has not been reported.

In this paper, we present the experimental evaluation of UOFDM-based VLC system using NI USRP platforms. Specifically, we first characterize the frequency-selective channel. Then, we obtain BER performance against the estimated signal-to-noise ratio (SNR) and confirm it through the comparison with theoretical results.

The rest of this paper is organized as follows. In Section II, we describe the U-OFDM system model under consideration. In Section III, we present practical implementation aspects such as channel estimation, frame detection and symbol timing estimation. In Section IV, we describe the experimental setup using USRPs. We further present measurement experimental results and comparisons with theoretical results. Finally, the paper is concluded in Section V.

\section{U-OFDM SIGNALLING MODEL}

The block diagram of U-OFDM is illustrated in Fig. 1. Let $S_{i}, i=1, \cdots, N / 2-1$ denote the $M$-QAM (quadrature amplitude modulation) symbols where $M$ is the modulation size and $N$ is the number of sub-carriers. In order to ensure that the output of inverse fast Fourier transform (IFFT) is real, Hermitian symmetry is imposed. The resulting signal is given by

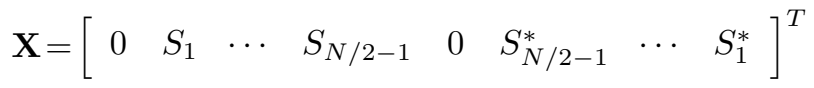

Let $\mathbf{X}[k]$ denote the $k^{\text {th }}$ element of $\mathbf{X}$. After IFFT, the time domain signal is expressed as $\mathbf{x}=\left[\begin{array}{lllll}\mathbf{x}[0] & \mathbf{x}[1] & \mathbf{x}[2] & \cdots & \mathbf{x}[N-1]\end{array}\right]^{T}$ whose $n^{\text {th }}$ element can be expressed as

$$
\mathbf{x}[n]=\frac{1}{\sqrt{N}} \sum_{k=0}^{N-1} \mathbf{X}[k] \exp (j 2 \pi n k / N), \quad n=0,1, \cdots, N-1
$$

A cyclic prefix $(\mathrm{CP})$ with a length of $N_{c p}$ is appended to $\mathrm{x}$ to prevent interference between OFDM symbols and to ensure the circular convolution with the channel. This makes possible the use of a single tap equalizer in frequency domain. After the addition of $\mathrm{CP}$, we have

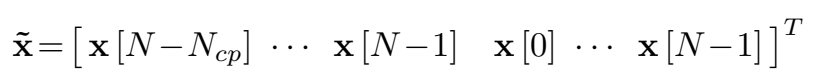

Due to Hermitian symmetry, $\tilde{\mathbf{x}}$ is real valued, but still bipolar. Based on U-OFDM structure, each time sample is encoded into a pair of samples. If $\tilde{\mathbf{x}}[n] \geq 0$, it will be encoded as $(\tilde{\mathbf{x}}[n], 0)$. On the other hand, if $\tilde{\mathbf{x}}[n]<0$, it will be encoded as $(0,-\tilde{\mathbf{x}}[n])$. First and second samples of each pair are assigned, respectively, to the positive and negative sub-frames which are denoted as $\tilde{\mathbf{x}}_{+}$and $\tilde{\mathbf{x}}_{-}$.

Let $p_{T}(t), P, T_{s}$ and $\delta(\cdot)$ denote transmit pulse shaping filter, average electrical transmit power, pulse duration and Dirac delta function, respectively. Furthermore, we introduce $\tilde{\mathbf{x}}_{ \pm}[n]$ to describe either $\tilde{\mathbf{x}}_{+}[n]$ or $\tilde{\mathbf{x}}_{-}[n]$ for a unified notation. The continuous time domain OFDM waveform can be expressed as

$$
x_{s \pm}(t)=A\left[\sqrt{P} \sum_{n=0}^{N+N_{c p}-1} \tilde{\mathbf{x}}_{ \pm}[n] \delta\left(t-n T_{s}\right)\right] \otimes p_{T}(t)+V_{D C}
$$

where $A$ is an amplification factor to mitigate the effects of path loss. Since it is likely that the first term in (4) can be still lower than LED turn on voltage, a DC bias $\left(V_{D C}\right)$ was further added. The signal goes through the optical channel. At the receiver side, a photodetector captures the light and converts into an electric current. The received electrical signal at the destination can be written as

$$
\tilde{y}_{ \pm}(t)=\left[R x_{s \pm}(t) \otimes l(t) \otimes h(t)+w_{ \pm}(t)\right] \otimes p_{R}(t)
$$




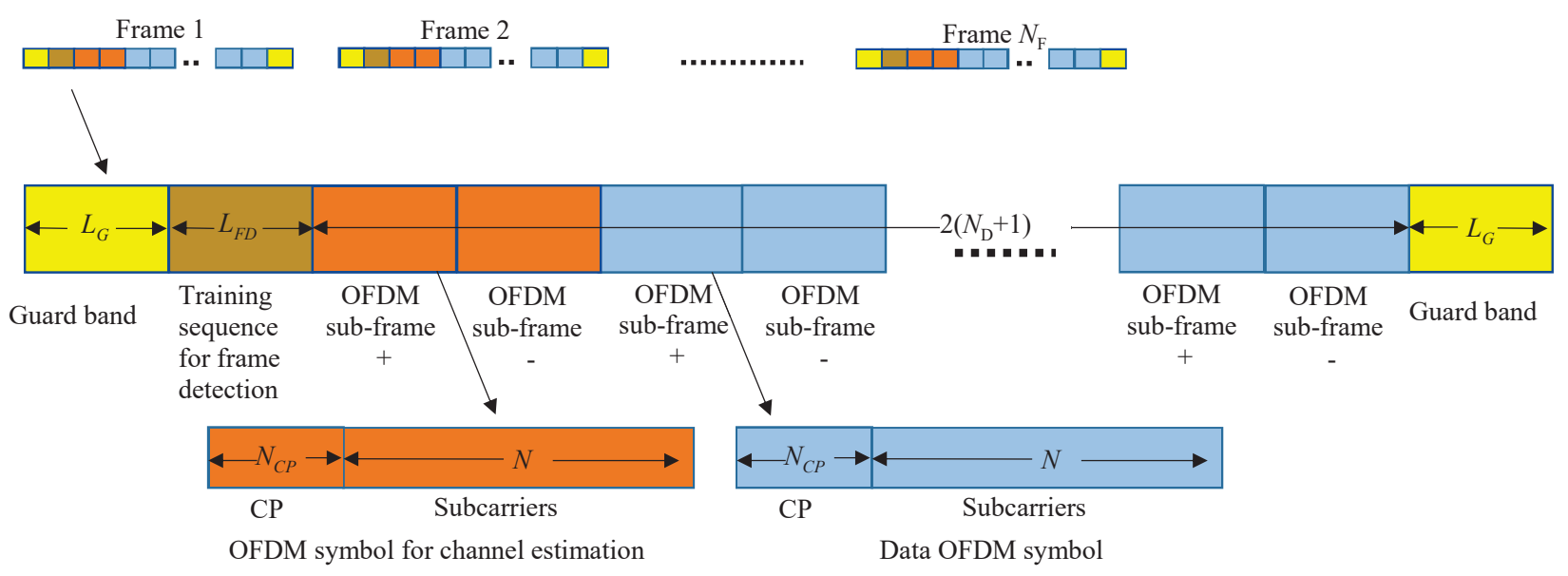

Fig. 2. Transmitted frame structure.

where $R$ is the photodetector responsivity, $l(t)$ is the LED impulse response, $h(t)$ is the propagation channel impulse response, $p_{R}(t)$ is the receive pulse shaping filter, and $w_{ \pm}(t)$ is the noise term. Replacing (4) in (5), we have

$$
\begin{aligned}
\tilde{y}_{ \pm}(t) & =\sqrt{P}\left[\sum_{n=0}^{N+N_{c p}-1} \tilde{x}_{ \pm}[n] \delta\left(t-n T_{s}\right)\right] \otimes h_{\text {eff }}(t) \\
& +V_{R_{D C}}(t)+w_{g_{ \pm}}(t)
\end{aligned}
$$

where we define $h_{e f f}(t)=A R p_{T}(t) \otimes l(t) \otimes h(t) \otimes p_{R}(t)$ as the effective channel impulse response incorporating the effects of propagation channel, LED response, and pulse shaping. In (6), we have further defined $w_{g_{ \pm}}(t)=w_{ \pm}(t) \otimes p_{R}(t)$ and $V_{R_{D C}}(t)=R V_{D C} \otimes l(t) \otimes h(t) \otimes p_{R}(t)$.

Let $w_{g_{ \pm}}(n), \tilde{y}_{ \pm}(n), V_{R_{D C}}(n)$ and $h_{\text {eff }}(n)$ denote, respectively, the sampled versions of $w_{g_{ \pm}}(t), \tilde{y}_{ \pm}(t), V_{R_{D C}}(t)$ and $h_{\text {eff }}(t)$. Further, noting that $V_{R_{D C}}(n)$ is the same for all samples, index $n$ can be dropped and $V_{R_{D C}}(n)$ can be written as $V_{R_{D C}}$. Therefore, the sampled version of $\tilde{y}_{ \pm}(t)$ can be expressed as

$$
\tilde{y}_{ \pm}(n)=\sqrt{P} \tilde{x}_{ \pm}(n) \otimes h_{e f f}(n)+V_{R_{D C}}+w_{g_{ \pm}}(n)
$$

After removing CP from $\tilde{y}_{ \pm}(n), n=0,1, \cdots, N+N_{\mathrm{CP}}-1$, we have

$$
y_{ \pm}(n)=\sqrt{P} x_{ \pm}(n) \otimes h_{e f f}(n)+V_{R_{D C}}+w_{g_{ \pm}}(n)
$$

From the descriptions of positive and negative U-OFDM subframes, it can be readily verified that $x(n)=x_{+}(n)-x_{-}(n)$. Therefore, we can write

$$
\begin{aligned}
y(n) & =y_{+}(n)-y_{-}(n) \\
& =\sqrt{P} x(n) \otimes h_{e f f}(n)+\bar{w}_{g}(n), n=0,1, \cdots, N-1
\end{aligned}
$$

In (9), $\bar{w}_{g}(n)=w_{g_{+}}(n)-w_{g_{-}}(n)$ is the noise term. After fast Fourier transform (FFT), the frequency domain signal at the $k^{\text {th }}$ subcarrier can be expressed as

$$
Y(k)=\sqrt{P} H_{e f f}(k) X(k)+\bar{W}_{g}(k), k=0,1, \cdots, N-1
$$

where $H_{\text {eff }}(k)$ and $\bar{W}_{g}(k)$ are, respectively, the $k^{\text {th }}$ element of $N$-point FFT of $h_{e f f}(n)$ and $\bar{w}_{g}(n)$.

For detection, we first equalize the received signal by using a single-tap equalizer. Mathematically speaking, we first obtain

$$
\widehat{X(k)}=\left(\sqrt{P} \widehat{H_{\text {eff }}(k)}\right)^{-1} Y(k), k=0,1, \cdots, N-1
$$

where $\widehat{H_{\text {eff }}(k)}$ is the estimated effective channel coefficient for the $k^{\text {th }}$ carrier (see Section III for details). Then, we perform maximum likelihood (ML) decoding, i.e.,

$$
\hat{X}(k)=\underset{X(k) \in \Omega}{\arg \min }\|\widehat{X(k)}-X(k)\|
$$

where $\|\cdot\|$ is the $L_{2}$ norm and $\Omega$ is the set of $M$-QAM symbols.

\section{Frame Detection, Symbol Timing AND SNR ESTIMATION}

The signal frame structure illustrated in Fig. 2. The start of each frame includes a guard band with a length of $L_{G}$ and a frame detection training sequence with a length of $L_{F D}$. This is then followed by the transmission of $2\left(N_{D}+1\right)$ OFDM sub-frames, i.e., positive and negative OFDM subframes following each other. Each OFDM sub-frame has a cyclic prefix with a length of $N_{c p}$ and data carriers with a length of $N$. The first two OFDM sub-frames carry the channel estimation training sequence while the other carry data ${ }^{1}$. Finally, a guard with a length of $L_{G}$ is inserted before the transmission of next frame. Therefore, a frame has a total length of $N_{\text {down }}=2 L_{G}+L_{F D}+2\left(N+N_{C P}\right)\left(N_{D}+1\right)$. We use root-raised-cosine (RRC) filter with a roll-off factor of $\beta$ for transmitter pulse shaping filter. After pulse shaping, the total number of samples in a transmitted frame is given by $N_{\text {up }}=N_{\text {down }} U$ where $U$ denotes the upsampling factor. In the following, we explain how we perform the symbol timing estimation, frame detection, and channel (and subsequent SNR) estimation.

\footnotetext{
${ }^{1}$ Due to Hermitian symmetry and unipolar coding, only $N / 2-1$ of $2 N$ carriers actually carry data while the others are loaded with zero.
} 
TABLE I

COMPONENTS AND RELATED SPECIFICATIONS

\begin{tabular}{|l|l|l|}
\hline \multicolumn{1}{|c|}{ Component } & \multicolumn{1}{c|}{ Brand and Model } & \multicolumn{1}{c|}{ Specifications } \\
\hline Amplifier & Mini-circuits ZHL-32A+ & 0.05 to $130 \mathrm{MHz}$ (Min 25 dB gain) \\
\hline NI USRP 2920 & Ettus LFTX and LFRX boards & 0 to $30 \mathrm{MHz}$ \\
\hline Bias-Tee & Mini-circuits ZFBT-GW+ & 0.1 to $6000 \mathrm{MHz}$ \\
\hline Photodetector & Thorlabs APD 130 A/M & 400 to $1000 \mathrm{~nm}$ \\
\hline LED & Lumileds LXA7-PW50 & High power white LED \\
\hline
\end{tabular}

First, we perform symbol timing which refers to the estimation of the sampling point of the symbol. We consider the maximum output energy method [16, Chapter. 5] which finds the sample point that maximizes the average received energy. Let $Y_{u p}(n), \quad n=1,2, \cdots, N_{\text {up }}$ denote the sampled received frame. Further let $\tau=1,2, \cdots, U$ denote the possible delay values. Then, we can write

$$
\hat{\tau}=\max _{\tau}\left|E\left[\left(Y_{u p}(n U+\tau)\right)^{2}\right]\right|, n=1,2, \cdots, N_{\text {down }}
$$

After symbol timing, frame detection is performed in order to resolve multiple symbol period delays. In other words, we determine the beginning of the frame. For frame detection, we use the training sequence $T_{\mathrm{FD}}(d), d=0, \cdots, L_{F D}-1$. By correlating the received signal with the known training sequence for frame detection that is placed at the header of the transmitted frame, the beginning of frame is found at the position of the autocorrelations peak. Mathematically speaking, the correlator output can be written as

$$
\hat{d}=\max _{n}\left|\sum_{d=0}^{L_{F D}-1} T_{\mathrm{FD}}(d) Y_{\text {down }}(n+d)\right|^{2}
$$

where $Y_{\text {down }}(n)=Y_{\text {up }}(n U+\hat{\tau}), \quad n=1,2, \cdots, N_{\text {down }} \quad$ is the down-sampled received frame.

For channel estimation, we use the training sequence $T_{C E}(i), i=1, \cdots, N / 2-1$ with unit power. Based on (10), the corresponding received frequency domain signal for the $k^{\text {th }}$ subcarrier can be written as

$$
Y(k)=H_{\text {eff }}(k) T(k)+\bar{W}_{g}(k), \quad k=0,2, \cdots, N-1
$$

Using zero-forcing estimator, the estimated channel gain at $k^{\text {th }}$ subcarrier, $k=0,1, \cdots, N-1$, can be written as

$$
\widehat{H_{\text {eff }}(k)}=Y(k) T^{-1}(k)
$$

It should be noted that the estimated effective channel gain will include the effects of channel and transmitter/receiver frontends, i.e., amplification gain, LED conversion loss, photodetector responsivity, and pulse shaping filters.

To measure the noise, we switch off the LED and measure the received signals. Assuming that $L$ samples are collected, the estimated noise power for the $k^{\text {th }}$ subcarrier can be written as

$$
\sigma_{k}^{2}=\frac{1}{L} \sum_{l=1}^{L}\left|Y_{l}(k)\right|^{2}
$$

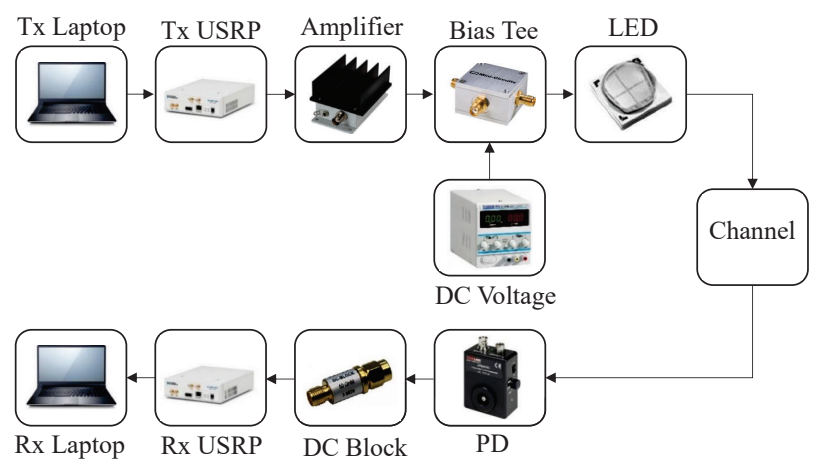

Fig. 3. Block diagram of the experimental set-up.

Since $\sigma_{n_{k}}^{2}$ remains the same for all subcarriers, we can drop index $k$ and write it as $\sigma_{n}^{2}$. Therefore, SNR at the $k^{\text {th }}$ subcarrier can be written as

$$
\widehat{S N R[k]}=\frac{\widehat{H e f f}(k)^{2}}{\sigma_{n}^{2}}
$$

\section{ExPerimental Setup and Measurement Results}

Fig. 3 illustrates the block diagram of our experimental setup. At the transmitter, LabVIEW software of the transmitter (source laptop) generates U-OFDM symbols. The generated data is transferred from the laptop via Ethernet cable to the modified NI 2920 USRP with LFTX daughter boards for digital to analog conversion (DAC). The output signal of the USRP is amplified by a power amplifier (Mini-circuits ZHL$32 \mathrm{~A}+$ ) to adjust the signal power for the input of Bias-Tee (Mini-circuits ZFBT-GW+). DC voltage is then added to shift the signal to the linear operation region of the LED. We use an LED (Lumileds LXA7-PW50) with turn-on voltage of $2.5 \mathrm{~V}$ and maximum forward voltage of $3.25 \mathrm{~V}$. At the receiver, we use an avalanche photodetector (APD) (Thorlabs APD $130 \mathrm{~A} / \mathrm{M}$ ). After the optical signal is converted into an electrical signal, DC component of the received signal is removed. This is then fed to the modified NI 2920 USRP with LFRX daughter board. U-OFDM demodulation is performed on LabVIEW software running on the destination laptop. The component types and specifications are summarized in Table I. The transmitter and receiver are placed apart from each other with a link distance of $d=1.2 \mathrm{~m}$. Average electrical transmit power $(P)$ is varied in the range of $0.1 \mathrm{~mW}$ and $3.6 \mathrm{~mW}$. 4 QAM is employed. The USRP sampling rate is $f_{s}=5 \times 10^{6}$ samples per second. We use an upsampling factor of $U=80$. We use RRC pulse shape with a roll-off factor of $\beta=0.5$. 
TABLE II

EXPERIMENTAL SET-UP PARAMETERS

\begin{tabular}{|l|l|c|}
\hline Link distance $(\mathrm{m})$ & $d$ & 1.2 \\
\hline Electrical Noise power $(\mathrm{dB})$ & $\sigma_{n}^{2}$ & -61.37 \\
\hline Average electrical transmitted power $(\mathrm{mW})$ & $P$ & 0.1 to 3.6 \\
\hline Modulation order & $M$ & 4 \\
\hline Number of data subcarriers & $N$ & 32 \\
\hline Length of cyclic prefix & $N_{c p}$ & 4 \\
\hline Training sequence length for frame detection & $L_{F D}$ & 80 \\
\hline Training sequence length for channel estimation & $L_{C E}$ & 15 \\
\hline Guard band length & $L_{G}$ & 6 \\
\hline Number of data OFDM symbols & $N_{D}$ & 368 \\
\hline Number of frames & $N_{F}$ & 60 \\
\hline Upsampling factor & $U$ & 80 \\
\hline USRP sampling rate (samples/sec) & $f_{s}$ & $5 \times 10^{6}$ \\
\hline RRC pulse shaping filter length & $L_{P S}$ & 80 \\
\hline Roll-off factor & $\beta$ & 0.5 \\
\hline
\end{tabular}

The pulse shape is truncated to the length of $L_{P S}=$ 80 samples. Each frame includes a guard band with a length of $L_{G}=6$, a frame detection training sequence with a length of $L_{F D}=80$, channel estimation training sequence with a length of $L_{C E}=15$, cyclic prefix with a length of $N_{c p}=4$ and data carriers with a length of $N=32$. For the convenience of the reader, all parameters are summarized in Table II.

Following the methodology described in Section III, we measured the effective channel gain per each subcarrier $H_{\text {eff }}(k)$ and illustrated in Fig. 4.a for different values of average electrical transmit power. It can be readily verified that the channel exhibits frequency-flat characteristics with very little fluctuation in frequency domain. This is rather expected since the transmitter and receiver have a line-of-sight configuration with a good alignment.

We further measured noise power based on $L=100$ noise samples for each subcarrier and, based on (17), we determined it as $\sigma_{n}^{2} \cong-61.37 \mathrm{~dB}$ for all subcarriers. Then, based on (18), we obtained the received SNR per each subcarrier and illustrated in Fig. 4.b.

For BER measurements, we transmitted $N_{F}=60$ frames. Each frame includes $N_{D}+1=369$ OFDM sub-frames, one of which is dedicated for channel estimation. Therefore, the total number of transmitted bits is given by $N_{F} N_{D}(N / 2-1) \log _{2} M=60 \cdot 368 \cdot 15 \cdot 2=6.624 \times 10^{5}$ bits. At the receiver side, these were demodulated based on the decision metric in (12) and compared with actual transmitted bits. The measured BER for each subcarrier is illustrated in Fig. 5. Averaging over $N / 2-1=15$ subcarriers, we obtain the measured average BER and plot it as well. As a benchmark, we further include the analytical BER expression. For square QAM constellations, an approximate expression of BER per

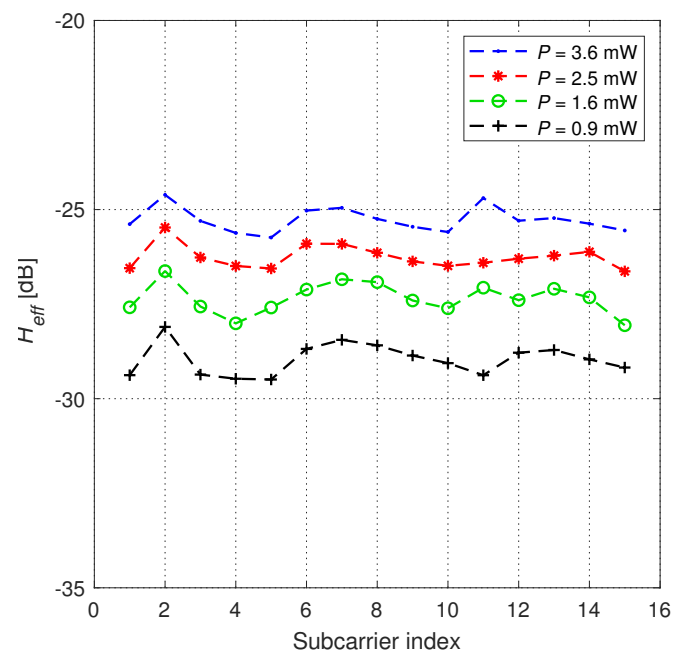

(a)

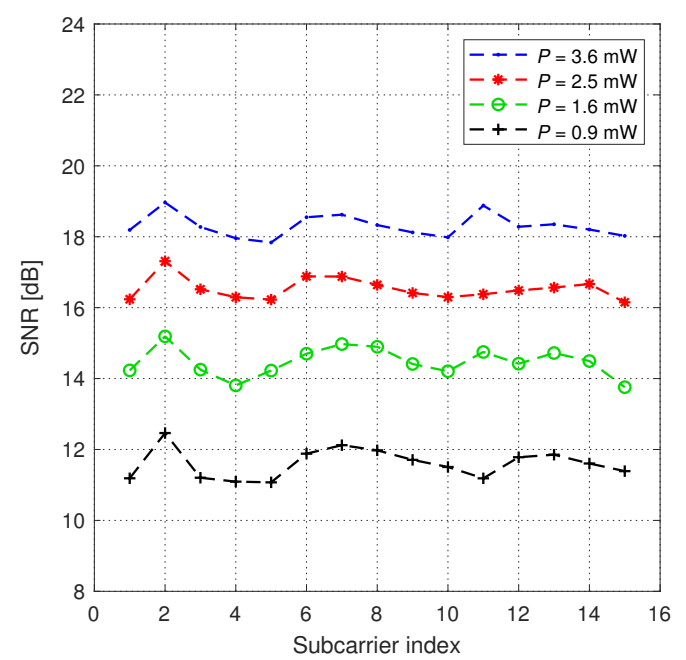

(b)

Fig. 4. (a) Effective channel per subcarrier and (b) SNR per subcarrier

subcarrier is given by [17]

$$
B E R[k] \cong \frac{2(\sqrt{M}-1)}{\sqrt{M} \log _{2} \sqrt{M}} Q\left(\sqrt{\frac{3 \mathrm{SNR}[k]}{2(M-1)}}\right)
$$

where $Q(\cdot)$ is the Gaussian Q-function. Averaging (19) over all data carriers, the average BER can be obtained by

$$
B E R=\frac{1}{N / 2-1} \sum_{k=1}^{N / 2-1} B E R[k]
$$

It is observed that the characteristics of theoretical and measured BER expressions match each other. There is around $2 \mathrm{~dB}$ shift between theoretical and experimental BER curves. This shift decreases as SNR increases. At low SNR values, frame synchronization and symbol timing experience higher estimation errors. At high SNR, the difference reduces to around $1.6 \mathrm{~dB}$ because of the better estimation quality. 


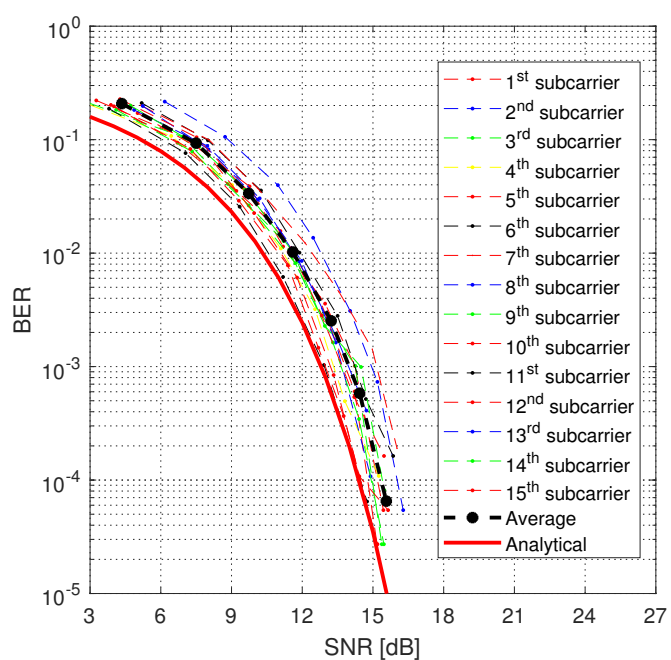

Fig. 5. Measured BER values and comparison with theoretical results.

\section{CONCLUSIONS}

U-OFDM has been proposed as a power-efficient multicarrier technique for high speed VLC systems. In this paper, we considered this promising optical OFDM technique and evaluated its performance using software defined platforms. Our experimental set-up was built upon the USRP from National Instruments customized with baseband cards and integrated with VLC front-ends. Through a measurement campaign, we first presented the frequency response of the effective channel including the effects of both propagation channel and frontends. Then, we measured the BER per subcarrier and obtained the overall BER. Experimental results were further confirmed through comparisons with theoretical results.

\section{REFERENCES}

[1] S. Dimitrov and H. Haas, Principles of LED Light Communications: Towards Networked Li-Fi. Cambridge, U.K.: Cambridge Univ. Press, 2015, ch. 2

[2] M. Uysal, C. Capsoni, Z. Ghassemlooy, A. Boucouvalas, and E. Udvary, Optical Wireless Communications: An Emerging Technology. Springer, 2016.
[3] P. H. Pathak, X. Feng, P. Hu, and P. Mohapatra, "Visible light communication, networking, and sensing: A survey, potential and challenges," IEEE Commun. Surveys Tuts., vol. 17, no. 4, pp. 2047-2077, 2015.

[4] J. Armstrong and B. J. C. Schmidt, "Comparison of asymmetrically clipped optical OFDM and DC-biased optical OFDM in AWGN," IEEE Commun. Lett., vol. 12, no. 5, pp. 343-345, May 2008.

[5] J. Armstrong and A. J. Lowery, "Power efficient optical OFDM," Electron Lett., vol. 42, no. 6, pp. 370-372, Mar. 2006.

[6] D. Tsonev, S. Sinanovic, and H. Haas, "Novel unipolar orthogonal frequency division multiplexing (U-OFDM) for optical wireless," in IEEE 75th Vehicular Technology Conference (VTC Spring), May 2012, pp. 1-5.

[7] A. J. Lowery, "Comparisons of spectrally-enhanced asymmetricallyclipped optical OFDM systems," Opt. Express, vol. 24, no. 4, pp. 39503966, Feb. 2016.

[8] J. Zhou and W. Zhang, "A comparative study of unipolar OFDM schemes in gaussian optical intensity channel," IEEE Trans. Commun., vol. 66, no. 4, pp. 1549-1564, Apr. 2018.

[9] D. Tsonev, S. Videv, and H. Haas, "Unlocking spectral efficiency in intensity modulation and direct detection systems," IEEE J. Sel. Areas Commun., vol. 33, no. 9, pp. 1758-1770, Sep. 2015.

[10] H. Durrani, J. Kasi, M. Sohail, M. Bokhari, and A. Kasi, "Implementation of unipolar OFDM based VLC transmission system under dimming constraint for high speed data through FPGA," J. Comput. Commun. Instrum.Engg., vol. 5, no. 1, pp. 14-17, 2018.

[11] O. Narmanlioglu, B. Turan, B. Kebapci, S. C. Ergen, and M. Uysal, "Poster: On-board camera video transmission over vehicular VLC," in IEEE Vehicular Networking Conference (VNC), Dec. 2016, pp. 1-2.

[12] Y. Qiao, H. Haas, and E. Knightly, "Demo: A software defined visible light communications system with WARP," in ACM Workshop on Visible Light Communication Systems, 2014.

[13] H. Elgala, R. Mesleh, and H. Haas, "Indoor broadcasting via white LEDs and OFDM," IEEE Trans. Consum. Electron, vol. 55, no. 3, pp. 1127-1134, Aug. 2009.

[14] W. Hussain, H. F. Ugurdag, and M. Uysal, "Software defined VLC system: Implementation and performance evaluation," in 4th International Workshop on Optical Wireless Communications (IWOW), Sep. 2015, pp. 117-121.

[15] L. Shi, W. Li, X. Zhang, Y. Zhang, G. Chen, and A. Vladimirescu, "Experimental 5G new radio integration with VLC", in 25th IEEE International Conference on Electronics, Circuits and Systems (ICECS), Dec. 2018, pp. 61-64.

[16] R. W. Heath, Jr, Introduction to Wireless Digital Communication: A Signal Processing Perspective. Englewood Cliffs, NJ, USA: PrenticeHall, Mar. 2017.

[17] K. Cho and D. Yoon, "On the general BER expression of one- and twodimensional amplitude modulations," IEEE Trans. Commun., vol. 50 , no. 7, pp. 1074-1080, Jul. 2002. 